\title{
Magnetic irreversibility and relaxation in assembly of ferromagnetic nanoparticles
}

\author{
R. Prozorov \\ Loomis Laboratory of Physics, University of Illinois at Urbana-Champaign, \\ 1110 W. Green St., Urbana, IL 61801, U.S.A. \\ Y. Yeshurun \\ Institute of Superconductivity, Department of Physics, Bar-Ilan University, \\ 52900 Ramat-Gan, Israel \\ T. Prozorov, A. Gedanken \\ Department of Chemistry, Bar-Ilan University, 52900 Ramat-Gan, Israel
}

(July 21, 1998)

\begin{abstract}
Measurements of the magnetic irreversibility line and time-logarithmic decay of the magnetization are described for three $\mathrm{Fe}_{2} \mathrm{O}_{3}$ samples composed of regular amorphous, acicular amorphous and crystalline nanoparticles. The relaxation rate is the largest and the irreversibility temperature is the lowest for the regular amorphous nanoparticles. The crystalline material exhibits the lowest relaxation rate and the largest irreversibility temperature. We develop a phenomenological model to explain the details of the experimental results. The main new aspect of the model is the dependence of the barrier for magnetic relaxation on the instantaneous magnetization and therefore on time. The time dependent barrier yields a natural explanation to the time-logarithmic decay of the magnetization. Interactions between particles as well as shape and crystalline magnetic anisotropies define a new energy scale that controls the magnetic irreversibility. Introducing this energy scale yields a self-consistent explanation of the experimental data.
\end{abstract}

\section{INTRODUCTION}

A ferromagnetic particle becomes monodomain if its size $d$ is reduced below a critical value $d_{c r} \sim 1-100 \mathrm{~nm}$, determined by the competition between dipole and exchange energieste. Below this critical size, the energy loss due to creation of magnetic domain walls (proportional to $d^{2}$ ) is larger than the gain due to disappearance of the dipole magnetic field energy (proportional to $d^{3}$ ). Such monodomain ferromagnetic particles can be viewed as large magnetic units, each having a magnetic moment of thousands Bohr magnetons. Usually neighboring particles are well separated $(10-20 \mathrm{~nm})$, and direct exchange between particles may be neglected. Thus, the magnetic properties of an assembly of nanoparticles are determined by the dipole field energy along with thermal and magnetic anisotropy energies (see e.g 3 d).

Experiments conducted on magnetic nanoparticles show irreversible magnetic behavior below the "irreversibility line" $T_{i r r}(H)$. In particular, the zero-field cooled (ZFC) and field cooled (FC) magnetization curves do not coincide, and magnetic hysteresis appears in $M$ vs. $H$ curves (see e. g. 10). Moreover, time-logarithmic magnetic relaxation, towards the thermodynamic equilibrium state, is observed below $T_{i r r}(H)$. Similar observations are reported here for three systems of $\mathrm{Fe}_{2} \mathrm{O}_{3}$ nanoparticles with different shape and crystalline magnetic anisotropies. These nanoparticle samples were prepared by a sonochemical method which produces "regular" amorphous nanoparticles11 13 . Sonochemical irradiation carried out in the presence of magnetic fieldresults in synthesis of acicular amorphous nanoparticles 13 . Annealing of amorphous particles leads to crystallization. In this manner we have prepared regular amorphous, acicular amorphous and crystalline ferromagnetic nanoparticles. This enables a study of the effect of shape and crystalline anisotropies on magnetic irreversibility and the relaxation rate. We find that, qualitatively, all three samples exhibit similar irreversible magnetic behavior. However, their irreversibility lines and relaxation rates differ significantly.

Irreversible magnetic behavior similar to that described here is also observed in other systems. A noticeable example is magnetic irreversibility in superconductors 14 16 . In such systems the origin for irreversibility is the interplay between thermal energy and some energy barrier, which prevents magnetic reorganization in those materials. The microscopic origin of the barrier, however, depends on the system. The magnetic irreversibility in nanoparticles is conventionally associated with the energy required for a particle moment reorientation, overcoming a barrier due to magnetic shape or crystalline anisotropy. It is important to note that the barrier is considored to be independent of the magnetic moment itsel 10.12 . 17 32 . In superconductors, magnetic irreversibility is due to the inevitable spatial fluctuation of the superconducting order parameter caused by defects, imperfections etc.; the barrier is the energy required to overcome the pinning due to this disorder.

An important concept in the theory of irreversible magnetic properties of superconductors, based on the work of Anderson 14 16, is that the effective barrier for magnetic relaxation increases with time. This is because 
the superconducting shielding current (proportional to the magnetization) decays with time, causing a decrease in the Lorentz force which drives the fluxons away of their positions. In a nice paper Lottis et al 33 have put forward similar arguments to study slow dynamics. They noticed the close analogy between ferromagnetic assemblies and superconductors. Analyzing the results of numerical computations they concluded that the decay of the demagnetizing field is the origin of what the "quasi-logarithmic" relaxation. Although the distribution of particle sizes may explain quasi-time-logarithmic relaxation in a limited time-interval, it is not necessary for the explanation of the experimentally observed timelogarithmic relaxation. This approach was later employed in other studies of the magnetic relaxation, for example, in thin magnetic films 34 .

In this work we adopt the concept of time varying barrier and derive the phenomenological model to explain magnetic irreversibility and logarithmic magnetic relaxation in nanoparticles. The physics for the time dependence of the barrier in nanoparticles is related to the fact that the effective barrier for reorientation of the magnetic moment of each nanoparticle depends on the internal magnetic field, which includes the average dipole field from surrounding nanoparticles. This average dipole field decreases with time due to the increase of randomness in the orientation of the magnetic moments of the surrounding nanoparticles. This, in turn, causes the increase of the effective barrier with time, yielding a natural explanation to the experimental observation of time-logarithmic relaxation, and a sample dependent irreversibility line and relaxation rate. Interactions between particles as well as shape and crystalline magnetic anisotropies define a new energy scale that controls the magnetic irreversibility. Introducing this energy scale yields a self-consistent explanation of the experimental data.

This article is organized in the following way. In Section II we describe the preparation of the three nanoparticle systems. We then describe our experimental results of irreversible magnetic properties at various temperatures, fields and times. In Section III we describe our phenomenological model and derive equations for the irreversibility line and the magnetic relaxation. In Section IV we compare the predictions of our model with the experimental results.

\section{EXPERIMENTAL}

\section{A. Sample preparation and characterization}

Three $\mathrm{Fe}_{2} \mathrm{O}_{3}$ samples composed of regular amorphous, acicular amorphous and crystalline namoparticles were prepared by a sonochemical method 1113 . For the ultrasound irradiation we used $V C-600$ Sonics and Materials sonicator with $\mathrm{Ti}$ horn at $20 \mathrm{kHz}$ and $100 \mathrm{~W} \cdot \mathrm{cm}^{-2}$. In
Table I we summarize their features. One molar solution of $\mathrm{Fe}(\mathrm{CO})_{5}$ in decaline was sonochemically irradiated for three hours in ambient pressure at $0{ }^{\circ} \mathrm{C}$. The powder obtained was centrifuged, washed repeatedly with dry pentane $(6-7$ times, $8500 \mathrm{rpm})$, and dried in vacuum at room temperature for three hours. The material obtained has been accumulated from $2-3$ sonications and the total amount of $\mathrm{Fe}_{2} \mathrm{O}_{3}$ was mixed to ensure the reliability of the results. Then, in order to remove organic residue, material was annealed in vacuum at $140-150{ }^{\circ} \mathrm{C}$ for three hours. Heating up to this temperature was necessary to evaporate residua of solvents, particularly decaline which has high a boiling point $\left(189-191^{\circ} \mathrm{C}\right)$. The amorphous nature of the particles is confirmed by X-ray diffraction, differential scanning calorimetry (DSC) analysis and electron-diffraction patterns at selected areas as shown in Figs. $1 a, 2 a$, and the inset to $3 a$, respectively. The absence of Bragg peaks in Fig. 1a demonstrates the absence of the long-range order in the atomic structure; the large endothermic peak in Fig. $2 a$ indicates an amorphous to crystalline transition at $\sim 400{ }^{\circ} \mathrm{C}$. The electron diffraction pattern of the inset to Fig. $3 a$ also confirm the amorphous nature of the particles. A typical particle size of $\sim 50 \mathrm{~nm}$ is inferred from the transmission electron micrography (TEM) picture of Fig. $3 a$.

Acicular amorphous particles have been prepared by performing sonication in external magnetic field of $7 \mathrm{kG}$ for three hours. The sonication has been carried out in the $0.25 \mathrm{M}$ solution of $\mathrm{Fe}(\mathrm{CO})_{5}$ in a flask open to air. We then repeat the wash and dry procedure as described above. The amorphous nature of the particles was confirmed by X-ray diffraction, DSC, and selected area electron-diffraction patterns as shown in Figs. 1b, $2 b$, and the inset to $3 b$, respectively. A typical particle length of $\sim 50 \mathrm{~nm}$ and diameter of $\sim 5 \mathrm{~nm}$ are inferred from TEM picture of Fig. $3 b$.

Heating of amorphous $\mathrm{Fe}_{2} \mathrm{O}_{3}$ up to $370-380{ }^{\circ} \mathrm{C}$ in ambient atmosphere for $3-4$ hours resulted in crystalline $\gamma-\mathrm{Fe}_{2} \mathrm{O}_{3}$ nanoparticles. The nature and the internal structure of the crystalline iron oxide were determined using the X-ray diffraction shown in Fig. 1c. The DSC data, Fig. $2 c$, do not show any endothermic peak. The TEM image of Fig. $3 c$ show particles of mean size of $\sim 200 \mathrm{~nm}$.

The second column in Table I summarizes typical particle size for the three samples. The third column includes the total surface area of the particles, as measured by BET absorption using $N_{2}$ gas as absorbent.

\section{B. Magnetic measurements procedure}

A Quantum Design MPMS SQUID magnetometer was used for all magnetic measurements reported here. The irreversibility line was determined from ZFC and FC magnetization measurements. Before taking a data point temperature was stabilized with $0.05 K$ accuracy and a 
30 sec pause was sustained. The temperature at which ZFC and FC merge for a constant field $H$ is defined as irreversibility temperature $T_{i r r}(H)$. We define the merging point using a criterion $\left|M_{F C}-M_{Z F C}\right| \approx 0.1 \mathrm{emu} / \mathrm{g}$.

The procedure for measurements of magnetic relaxation at different temperatures is as follows: The sample is cooled in $H=2$ Tesla from a room temperature (larger than $T_{i r r}(2$ Tesla $)$ ) to a target temperature $T$, the magnetic field is then reduced to $500 G$ and the magnetic moment is measured for approximately two hours. The first data point is taken approximately two minutes after the field change.

The field dependence of the magnetic relaxation rate is measured at $T=20 \mathrm{~K}$. At this temperature the field is ramped up to $H=2$ Tesla and reduced back to a target field $H$, from where the measurements start. The same has been repeated for negative field $H=-2$ Tesla with consequent a increase of the magnetic field to a target value and measurements of the magnetic relaxation.

\section{Results}

The experimental results in this section are organized as follows: we first show $M(T, H=$ constant) data, and related measurements of magnetic relaxation at different temperatures. From the merging point of the ZFC and FC magnetization curves we extract the irreversibility line for the three samples. From the relaxation measurements we deduce the relaxation rate, as a function of temperature, for the three samples. We then present measurements of magnetization loops $M(T=$ constant, $H)$ and magnetic relaxation at different values of external field. The relaxation rate, as a function of field, is then deduced for the three samples.

Fig. 4 exhibits typical results of ZFC-FC magnetization curves and magnetic relaxation at $500 \mathrm{G}$ for the sample composed of amorphous round nanoparticles. The vertical lines of open circles in Fig. 4 depict the relaxation measurements at different temperatures. The vertical arrow indicates the direction of the time increase. The magnetic moment relaxes towards the equilibrium moment $M_{r e v}$, determined by the FC curve. In the inset to Fig. 4 we zoom out at the ZFC-FC curves and indicate by an arrow, the experimental definition of $T_{i r r}$.

The magnetic relaxation data of Fig. 4 are re-plotted in Fig. 5 as a function of time. The solid lines in Fig. 5 are linear fits for $M \propto \ln (t)$. A qualitatively similar time-logarithmic decay is also observed in the other two samples. Quantitative differences will be discussed below.

We define the "normalized relaxation rate" $R=$ $|\partial M / \partial \ln (t)| / M_{c}$, i. e., the logarithmic slope of the relaxation curve normalized by the magnitude of the irreversible magnetization at which the relaxation starts, $M_{c}=M_{0}-M_{r e v}$. Here $M_{0}$ is the initial value of the total magnetic moment and $M_{r e v}$ is the magnetic mo- ment corresponding to a field cooling in $500 \mathrm{G}$. Fig. 6 summarizes the values of $R$ as a function of temperature, for the three samples. At low enough temperatures, $R$ is the lowest for the crystalline sample, intermediate for the acicular amorphous sample and the largest for the regular amorphous sample. Note, that at higher temperatures it looks as if $R(T)$ curves will cross. This is due to a large difference in the absolute values of $T_{i r r}(90 \mathrm{~K}$, $162 \mathrm{~K}$ and $216 \mathrm{~K}$ at $500 \mathrm{G}$ for regular amorphous, acicular amorphous and crystalline, respectively). As shown in Fig. 16, $M(T)$ curves scale with $T_{i r r}$ and, therefore, in the inset to Fig. 6 we plot $R$ vs. $T / T_{i r r}$. In this presentation, the whole $R\left(T / T_{i r r}\right)$ curve of the crystalline sample is lower than that of the acicular amorphous sample and both are lower than the $R$ curve of the regular amorphous sample.

In Fig. 7 we compare the irreversibility lines for the three samples. The largest irreversibility is found in a crystalline sample, intermediate in the sample with acicular particles and the lowest in the regular amorphous sample. We explain these observations below.

Magnetic irreversibility below $T_{i r r}$ is also demonstrated by measuring the magnetization loops $M(H)$. As an example, we show in Fig. $8 M(H)$ for the amorphous nanoparticles at $T=5$ and $100 K$. Magnetic hysteresis is apparent at $5 K$, whereas the behavior is purely reversible at $100 \mathrm{~K}$.

The relaxation at different values of the external magnetic field is shown in Fig. 9. The vertical lines represent $M(t)$ curves shown along with the standard magnetization loop. The field dependencies of the relaxation rates for our samples are shown in Fig.10. There is an apparent change in $R$ between low and high fields. At lower fields $R$ is the largest in an amorphous sample, whereas at large fields the relaxation rate in an amorphous sample is the lowest.

\section{MAGNETIC RELAXATION IN THE ASSEMBLY OF NANOPARTICLES}

\section{A. Time dependent effective barrier for magnetic reorganization}

Magnetic relaxation is a distinct feature of systems with interacting particles, far from thermodynamic equilibrium. In an assembly of ferromagnetic nanoparticles, the elementary process of a change in the magnetization is the rotation of the magnetic moment of a nanoparticle (or cluster of such magnetic moments). In the following we assume that the magnetic anisotropy of each nanoparticle is strong enough to utilize an Ising-like model, i. e., the magnetic moment of each particle is aligned only along the anisotropy axis. In Fig. $11 a$ we illustrate schematically the orientation of the elementary magnetic moments of several of such nanoparticles. The full arrows represent the size and direction of each magnetic 
moment. The experimentally measured magnetic moment is determined by the sum of the projections of each individual particle's moment on the direction of the external magnetic field. Note that the directions of the easy axes are randomly distributed. For such a system, the energy $W$ of each magnetic nanoparticle, neglecting for the moment the interparticle interactions, varies with the angle as 21 .

$$
W=K V \sin ^{2}(\varphi-\theta)-M_{p} H \cos (\varphi)
$$

Here $\theta$ is the angle between the easy axis $\vec{K}$ and the external magnetic field $\vec{H}$, and $\varphi$ is the angle between the particle magnetic moment $\vec{M}_{p}$ and the external field. In order to have any magnetic irreversibility and relaxation, the $K V$ term in Eq. 1 must be larger than the $M_{p} H$ term and we will consider this limiting case. The reduced energy $W / K V$ of Eq. 11 is plotted in Fig. 12 as a function of the angle $\varphi$ for two different fields $H_{1}=2.5 \mathrm{KV} / \mathrm{M}_{p}$ (bold) and $H_{2}=0.5 \mathrm{KV} / M_{p}$ (light). Since the magnetic anisotropy has no preferable direction, there are two minima in the angular dependence of the energy, as shown in Fig. 12. The external magnetic field fixes the direction of the lowest minima. We denote by $U_{12}$ the barrier for reorientation from the lowest minima $\left(W_{1}\right)$ to the other minima $\left(W_{2}\right)$. The backward reorientation requires overcoming the energy barrier $U_{21}$.

In order to take inter-particle interactions into account we view the field $H$ in Eq. 1 as the internal magnetic field, which is the sum of the external field and the dipole field from the surrounding nanoparticles. This local magnetic field depends on the directions of neighboring magnetic moments35 36 . Since the magnetic moment is a statistical average of those moments, the local field depends, on the average, on the total magnetic moment. This induces a feedback mechanism: each reorientation of an individual nanoparticle decreases the total magnetic moment. This is illustrated in Figs. 11a. and 11b. Figure $11 a$ represents a snapshot of a field-cooled system of nanoparticles in which most of the individual magnetic moments are favorably oriented in a direction such that their projections are along the external field. After a field decrease, as a result of thermal fluctuations, some magnetic moments reorient so that their projection is anti-parallel to the external field. The open arrows in Fig. $11 b$ represent those reoriented moments.

Since the local dipole field decreases during this process, the average barrier $U_{12}$ increases. As indicated in the Introduction, an increase of the barrier with time is a characteristic of other irreversible systems, such as type-II superconductors in the process of magnetic flux creep.

The dynamics resulting from such a scenario is sketched Fig. 13. Immediately after reducing the magnetic field, individual magnetic moments are still along the direction of the external field, i. e., in minima $W_{1}$ of Fig. 13, as depicted by the population of the black dots. During the relaxation process magnetic moments flip to the minimum $W_{2}$ in the figure. Since, as discussed above, this barrier depends on the total magnetic moment via dipole fields, it will increase with time as shown in the figure, with dipole fields working on the average against the external field. The total magnetic moment along the magnetic field is thus decreased, as sketched in Fig. $11 b$.

\section{B. Equations of magnetic relaxation}

In a realistic sample, the directions of easy axes are randomly distributed, the particles cannot physically rotate (e. g., in a dense powder of ferromagnetic nanoparticles), and dipole interactions are strong. We will model this situation as outlined below.

Any given particle $i$ in Fig. 11 has an anisotropy axis at a fixed angle $\theta_{i}$ relative to the external magnetic field. The magnetic moment of this particle is then oriented at an angle $\varphi_{i}$ to the field. This angle is defined by the non-local energy minimization, due to dipole fields of the surrounding. It is important to note that each particle interacts with a local magnetic field $H_{i}$ which is the result of a vector sum of the external and dipole fields. At small enough external field and large enough anisotropy $\varphi_{i}$ may have two values: $\varphi_{i} \approx \theta_{i}$ or $\varphi_{i} \approx$ $\theta_{i}+\pi$, which leads to the situation described in Fig. 12, with two energy minima at $W_{1}^{i} \approx-M_{p} H_{i} \cos \left(\theta_{i}\right)$ and $W_{2}^{i} \approx M_{p} H_{i} \cos \left(\theta_{i}\right)$. Thermal fluctuations may force particle moment in the minima $W_{1}^{i}$ to change its direction to another minima $W_{2}^{i}$ and vise versa. The $W_{1}^{i} \rightarrow W_{2}^{i}$ rotation requires overcoming a barrier $U_{12}^{i}$, and a barrier $U_{12}^{i}$ for backward rotation, see Eqs. 16 and 17 of the Appendix, respectively. We then assume that the field $H_{i}$ can be represented as a simple sum of the external field $H$ and the collinear to $H$ dipole field $H_{d}$ (i.e. independent of $\theta_{i}$ ). The amplitude of a dipole field $H_{d}$ at any given site depends upon orientations of the moments of the surrounding particles. If those orientations are totally random (minima $W_{1}$ and $W_{2}$ are equally occupied) the dipole field is small, whereas if all surrounding particles are in one of the minima the resulting dipole field is maximal. From this simple analysis, we conclude that the magnitude of a dipole field depends upon the total magnetic moment $M$.

Considering the balance of forward and backward rotations, and averaging over the volume of the sample, we show in the Appendix that magnetic relaxation is described by a differentiple equation similar to that derived for superconductors 15 . 16 :

$$
\frac{\partial M}{\partial t}=-A M_{c} \exp \left(-\frac{U}{T}\right)
$$

where $A$ is an attempt frequency and $U$ is an effective barrier for magnetic relaxation given by

$$
U=U_{0}\left(1-\frac{M}{M_{0}}\right)
$$


where

$$
U_{0}=2 K V+4 M_{p}\left(H-\gamma M_{r e v}\right) / \pi
$$

and $M_{0}=\frac{1}{\gamma}\left(\frac{\pi K V}{2 M_{p}}+H-\gamma M_{\text {rev }}\right)$. Here $\gamma$ is the constant accounting for the strength of the dipole-dipole interactions, $M_{p}$ is the magnetic moment of an individual particle, $K$ is the anisotropy constant and $V$ is the particle volume. Apparently, as $\gamma \rightarrow 0$ the energy barrier $U \rightarrow U_{0}$, thus $U_{0}$ is the barrier in the assembly of noninteracting particles. It is worth noting that in our model the barrier $U$ depends on the magnetic moment in the same way as that used by Anderson 1416 for a description of magnetic relaxation in superconductors.

In the following we analyze magnetic relaxation described by those equations.

If the barrier for a particle moment reorientation does not depend on the total magnetic moment, i.e., $\gamma=0$ and $U=U_{0}$, direct integration of Eq. 2 yields:

$$
M=M_{c} \exp (-t / \tau)
$$

where $M_{c}$ is the initial irreversible magnetization and $\tau=\exp \left(U_{0} / T\right) / A$ is the macroscopic characteristic relaxation time. This result is very similar to that derived in early works for_classical Néel's superparamagnetic relaxation, see e.g.,37. This exponential decay is observed experimentally, for example in the work of Wegrowe et al. 19 on a single nano-wire.

If interactions are not negligible, Eq. 2 may be rewritten in dimensionless form:

$$
\frac{\partial u}{\partial \widetilde{\tau}}=-\exp (-u)
$$

where $u=U / T$ and $\widetilde{\tau}=t / \widetilde{t}$ with

$$
\widetilde{t}=\frac{M_{0}}{M_{c}} \frac{T}{U_{0}} \frac{1}{A}=\frac{\pi T}{4 \gamma A M_{p} M_{c}}=\frac{T}{A \Theta}
$$

where we introduced a new energy scale $\Theta$, which, as we show below, determines the relaxation process and the irreversibility line:

$$
\Theta=M_{c} U_{0} / M_{0}=4 \gamma M_{c} M_{p} / \pi
$$

This energy is directly related to the strength of the interparticle interactions.

Solving Eq. 6 we obtain

$$
u=u_{c}+\ln \left(1+\frac{t}{t_{0}}\right)
$$

where $u_{c}=U_{c} / T$ is the reduced effective energy barrier at $t=0$, the time when the relaxation starts. The normalization time $t_{0}$ is given by

$$
t_{0}=\widetilde{t} \exp \left(\frac{U_{c}}{T}\right)=\frac{T}{A \Theta} \exp \left(\frac{U_{0}-\Theta}{T}\right)
$$

Now, using Eqs. 3 and 9 we get the time evolution of the magnetic moment:

$$
M(t)=M_{c}\left(1-\frac{T}{\Theta} \ln \left(1+\frac{t}{t_{0}}\right)\right)
$$

Normalized relaxation rate $R \equiv|\partial M / \partial \ln (t)| / M_{c}$ is given by:

$$
R=\frac{T}{\Theta} \frac{t}{t_{0}+t}
$$

As we will see below, experiment shows that $t_{0}<1 \mathrm{sec}$. In our measurements typical time window $\Delta t \approx 100 \mathrm{sec}$, therefore we can assume $t \gg t_{0}$ and Eq. 12 predicts that the relaxation rate saturates at $R=T / \Theta$. Thus, measurements of the normalized relaxation rate can provide direct estimate of the energy scale $\Theta$ governing the relaxation process.

\section{Irreversibility temperature}

The irreversibility temperature $T_{i r r}$ of the assembly of magnetic nanoparticles is defined by the condition $M\left(\Delta t, T_{i r r}\right)=\Delta M$. Here $\Delta M$ is the smallest measured magnetic moment and $\Delta t$ is the time window of the experiment. Using Eq. 11 we obtain:

$$
T_{i r r}=\Theta \frac{1-\Delta M / M_{c}}{\ln \left(1+\Delta t / t_{0}\right)} \approx \frac{\Theta}{\ln \left(1+\Delta t / t_{0}\right)}
$$

thus we can estimate the characteristic time $t_{0}$ from measurements of $T_{i r r}$, because the energy $\Theta$ can be determined separately from the measurements of the relaxation rate $R \approx T / \Theta$. On the other hand the irreversibility line $T_{i r r}(H)$ gives the field dependence of $\Theta$. The latter may be obtained also from $R(H)$ measurements. Thus measurements of $T_{i r r}(H)$ and $R(H)$ in different samples provide a verification of our model on self-consistency.

It is interesting to note that the expression for $T_{i r r}, \mathrm{Eq}$. 13 , is typical for the blocking temperature of individual non-interacting particles, which is obtained from Eq. 5:

$$
T_{i r r}^{0}=\frac{U_{0}}{\ln \left(\Delta t / t^{*}\right)}
$$

where $t^{*}=1 /\left(A \ln \left|M_{c} / \Delta M\right|\right)$ is the characteristic time and $U_{0}$ is given by Eq. 4 . Energy $U_{0}$ is proportional to $K V$ for non-interacting nanoparticles, but it is reduced by a term proportional to $\gamma$ due to interparticle interactions. This is in agreement with previous works where "static" modifications of the barrier for relaxation were considered12723.38. Irreversibility temperature, Eq. 14 approaches 0 when $\Delta M \rightarrow 0$, and so does $T_{i r r}^{0}$. This reveals an important difference in the physics of the irreversibility line in interacting and non-interacting particles. In the former, there is a true irreversibility in 
the limit $\Delta M \rightarrow 0$ associated with freezing of magnetic moments due inter-particle interactions. In the case of non-interacting nanoparticles, the apparent irreversibility is due to experimental limitations (finite sensitivity, e.g., $\Delta M)$. It is important to stress that this is true only on a macroscopic time scale $\Delta t \gg t^{*}$, such as relaxation or $M(T)$ measurements. If, however, $\Delta t<t^{*}$ is realized, for example in Mössbauer measurements, one may detect the irreversibility temperature according to Eq. $1412.24,39$

We also note that in any case $T_{i r r}$ is a dynamic crossover from reversible to irreversible state and is defined for a particular experimental time window $\Delta t$.

In the following section we compare our experimental observations with the model developed above.

\section{DISCUSSION}

Our phenomenological model provides a description of the irreversible magnetic behavior in the assembly of ferromagnetic nanoparticles. In particular, the model predicts the time-logarithmic decay of the magnetization, see Eq. 11. Also, Eqs. 12 and 13 relate both the irreversibility line and the relaxation rate to a single parameter $\Theta=4 \gamma M_{c} M_{p} / \pi$.

The magnetic relaxation data of Fig. 5 reveal, indeed, time-logarithmic relaxation. Fitting these data to Eq. 11 yields the parameters of $M_{c}$ and $\Theta$. In Fig. 14 we plot the derived energy $\Theta$ as a function of temperature for the three samples and find that $\Theta$ is the largest for a crystalline sample, intermediate for an acicular amorphous and the lowest for a regular amorphous sample. The straightforward explanation is that in a crystalline sample both $\gamma$ and $M_{p}$ are the largest; in an acicular amorphous sample $M_{p}$ is of the same order as in regular amorphous, but $\gamma$ is much larger due to shape anisotropy.

Similarly, we derive the magnetic field dependence of $\Theta$ from the data of Fig. 9 and plot $\Theta(H)$ for three samples in Fig. 15. We note that $M_{p}$ and $\gamma$ should not depend on magnetic field. It is therefore expected that the field dependence of $\Theta$ is determined by the field dependence of $M_{c} \approx M_{s}-M_{\text {rev }}(H)$, which decreases with field, Fig. 9. Figure 15 shows the agreement with this observation. The weak increase of $\Theta$ with temperature, Fig. 14, may be related to some non-linear dependence of barrier $U$ on the magnetic moment.

Independent estimations of $\Theta$ are derived from $T_{i r r}$ of Fig. 7 using Eq. 13. Comparing Fig. 7 and Fig. 15 we get (for three samples) $\Theta / T_{i r r} \approx 4-6$. Thus, $t_{0} \approx 0.05-0.5 \mathrm{sec}$. Note that these values of $t_{0}$ are much larger than the "microscopic" values predicted by Néel37, simply because they reflect collective behavior of the whole assembly controlled by the effective barrier $\Theta$, see Eq.10, and not a single particle barrier $K V$.

Let us now compare the irreversibility lines of different samples, Fig. 7. In most parts of this diagram the region of the irreversible behavior is the largest for a crystalline sample. The amorphous sample containing acicular particles occupies the intermediate space and the amorphous sample embraces the smallest space in this $T-H$ phase diagram. Such behavior is naturally explained in terms of a strength of inter-particle interactions, which are the smallest in the case of a regular amorphous sample, intermediate for an acicular amorphous sample (due to shape anisotropy) and the largest for a crystalline sample due to crystalline anisotropy. Also, highest irreversibility temperature of crystalline sample is understood on the basis of its largest particle size.

\section{SUMMARY AND CONCLUSIONS}

We presented measurements of irreversible magnetization as a function of temperature, time and magnetic field in three types of ferromagnetic nanoparticles: regular amorphous, acicular amorphous and crystalline nanoparticles. The results are interpreted using a developed phenomenological approach based on the assumption that the barrier for magnetic moment reorientation depends on the total magnetic moment via dipole fields. This explains the time-logarithmic magnetic relaxation governed by the energy scale $\Theta$ related to interparticle interaction. Values of $\Theta$ found from measurements of the irreversibility line and the relaxation rate are in perfect agreement, implying validity of our model.

Acknowledgments: We thank Y. Rabin and I. Kanter for valuable discussions. This work was partially supported by The Israel Science Foundations and the Heinrich Hertz Minerva Center for High Temperature Superconductivity. Y.Y. acknowledges support from the German Israeli Foundation (G.I.F). R. P. acknowledges support from the Clore Foundations. 


\section{APPENDIX: EFFECTIVE BARRIER FOR MAGNETIC RELAXATION AND EQUATION FOR TIME EVOLUTION OF THE MAGNETIC MOMENT}

Here we consider in detail the model outlined in the text. We assume that magnetic moment $M_{p}$ of any given particle $i$ can be in one of the two possible energy minima: $W_{1}^{i} \approx-M_{p} H_{i} \cos \left(\theta_{i}\right)$ or $W_{2}^{i} \approx M_{p} H_{i} \cos \left(\theta_{i}\right)$. These minima are separated by the barrier of height $\sim K V+M_{p} H_{i} \sin \left(\theta_{i}\right)$. In the presence of thermal fluctuations, a particle moment sitting in the minima $W_{1}^{i}$ can spontaneously change its direction to the next minima $W_{2}^{i}$. The energy barrier for such reorientation is

$$
U_{12}^{i}=K V+M_{p} H_{i}\left(\sin \left(\theta_{i}\right)+\cos \left(\theta_{i}\right)\right)
$$

The backward rotation is also possible and requires overcoming the barrier:

$$
U_{21}^{i}=K V+M_{p} H_{i}\left(\sin \left(\theta_{i}\right)-\cos \left(\theta_{i}\right)\right)
$$

From this point on one can conduct a self-consistent statistical average over angles $\varphi_{i}\left(H_{i}, t, \theta_{i}\right)$ in order to evaluate the resulting magnetic moment $M$ of the system. On the other hand we may try to simplify the problem assuming that the internal field $H_{i}$ can be represented as a simple sum of the external field $H$ and the collinear to it dipole field $H_{d}$ (i.e. independent of $\theta_{i}$ ). If all easy axes are randomly distributed the average barrier for flux reorientation is then given by

$$
U_{k} \equiv\left\langle U_{i}\right\rangle_{k}=\frac{1}{N_{k}} \sum_{i=1}^{N_{k}} U_{i} \approx \frac{2}{\pi} \int_{0}^{\pi / 2} U(\theta) d \theta
$$

where $k=12$ or 21 denotes particle's moment flipping from the minima $W_{1}$ to the minima $W_{2}$, or backward, respectively. Using Eq. 15 and Eq. 16 we find

$$
\begin{aligned}
& U_{12}=K V+4 M_{p} H_{i} / \pi \\
& U_{21}=K V
\end{aligned}
$$

Let us now consider a situation where temperature is higher than irreversibility temperature and system is at thermal equilibrium. The number of particles jumping per unit time from one minima to another is proportional to $N_{k} \exp \left(-U_{k} / T\right)$. The condition for equilibrium is

$$
N_{1} e^{-\frac{U_{12}}{T}}=N_{2} e^{-\frac{U_{21}}{T}}
$$

Thus

$$
N_{2}=N_{1} \exp \left(-\frac{U_{12}-U_{21}}{T}\right)=N_{1} \exp \left(-\frac{4 M_{p} H_{i}}{\pi T}\right)
$$

It is clear that the difference $n=N_{1}-N_{2}$ determines the resulting magnetic moment of a system. If the total number of particles in the system is $N$ difference, $n$ is

$$
n=N_{1}-N_{2}=N \frac{1-\exp \left(-\frac{4 M_{p} H_{i}}{\pi T}\right)}{1+\exp \left(-\frac{4 M_{p} H_{i}}{\pi T}\right)}=N \tanh \left(\frac{2 M_{p} H_{i}}{\pi T}\right)
$$

The total reversible magnetic moment then is

$$
M_{r e v} \approx M_{p} n=M_{p} N \tanh \left(\frac{2 M_{p} H_{i}}{\pi T}\right)
$$

This formula is similar to the expression for the Ising superparamagnet and simply reflects the two-state nature of our model 37,40. The difference is, however, that the physical magnetic field is the total (external + dipole) field $H_{i}$.

Dipole field $H_{d}$ at any given site depends upon orientations of the moments of the surrounding particles. If those orientations are totally random (minima $W_{1}$ and $W_{2}$ are equally occupied) the dipole field is small, whereas if all surrounding particles are situated in one of the minima the resulting dipole field is maximal. From this simple picture, we conclude that the magnitude of a dipole field depends upon the total magnetic moment of a sample $M_{r e v}+M$, where $M_{r e v}$ is given by Eq. 20 and $M$ is the irreversible, time dependent contribution to the total magnetic moment resulting from the finite relaxation time needed for a system to equilibrate. Therefore, we may write $H_{i}=H-\gamma\left(M_{r e v}+M\right)$. Here $\gamma$ is the coefficient accounting for the contribution of dipole fields. Now we can obtain the equation for reversible magnetization from Eq.20:

$$
M_{\text {rev }} \approx M_{S} \frac{\tanh \left(\frac{2 M_{p} H}{\pi T}\right)}{1+\gamma \frac{2 M_{p} M_{S}}{\pi T}}
$$

where $M_{S}=M_{p} N$. We note that this formula is valid at small enough fields $2 M_{p} H / \pi<T$ when particle moments are almost locked along the easy axes and small enough interactions (i.e. $H>\gamma M_{\text {rev }}$ ) . The important result is that reversible magnetization decreases as the inter-particles interaction increases. Interestingly, Eq. 21 provides a good description of the experimental data.

Thus, the barriers for moment reorientation in Eq. 17 and Eq. 18 can be re-written as

$$
\begin{aligned}
& U_{1}=K V+4 M_{p}\left(H-\gamma\left(M_{r e v}+M\right)\right) / \pi \\
& U_{2}=K V
\end{aligned}
$$

We shall now consider direct and backward moment rotation processes in a non equilibrium state. As above, we denote by $N_{1}$ and $N_{2}$ number of moments in energy minima 1 and 2 , respectively. The total number of particles in the system is $N=N_{1}+N_{2}$. The magnetic moment is proportional to the difference $n=N_{1}-N_{2}$. During small time $\delta t$ this difference changes as

$$
\delta n=\left(N_{1} \exp \left(-\frac{U_{1}}{T}\right)-N_{2} \exp \left(-\frac{U_{2}}{T}\right)\right) \delta t
$$


Using simple algebra and above relationships between $N_{1}, N_{2}, n$, and $N$, we get

$\frac{\delta n}{\delta t}=-\exp \left(\frac{U_{1}+U_{2}}{T}\right)\left(n \cosh \left(\frac{U_{1}-U_{2}}{T}\right)+N \sinh \left(\frac{U_{1}}{T}\right.\right.$

From this we arrive to a non-linear differential equation governing process of magnetic relaxation not too close to equilibrium:

$\frac{d M}{d t} \approx-A \exp \left(\frac{U_{1}+U_{2}}{T}\right)\left(\left(M_{r e v}+M\right) \cosh \left(\frac{U_{1}-U_{2}}{T}\right)+\right.$

where $A$ is a constant measured in $\sec ^{-1}$ and having meaning of attempt frequency.

Eq. 26 can be simplified considering magnetic relaxation not too close to equilibrium and retaining our assumption that anisotropy contribution to the magnetic energy is much larger than that of magnetic field (both conditions are better satisfied at low fields). In this case, Eq. 26 may be approximated in a reduced form:

$$
\frac{\partial M}{\partial t}=-A M_{c} \exp (-U / T)
$$

where $M_{c}$ is the total magnetic moment at the beginning of relaxation and $U$ is the effective barrier:

$U=2 K V+4 M_{p}\left(H-\gamma M_{r e v}-\gamma M\right) / \pi=U_{0}\left(1-\frac{M}{M_{0}}\right)$

where $U_{0}=K V+4 M_{p}\left(H-\gamma M_{\text {rev }}\right) / \pi$ and $M_{0}=$ $\frac{1}{\gamma}\left(\frac{\pi K V}{2 M_{p}}+H-\gamma M_{r e v}\right)$

We reiterate that Eq. 27 is valid only in the case when the magnetic anisotropy is large and magnetic moment is far from equilibrium. Close to equilibrium, one ought to consider Eq. 26.

${ }^{1}$ J. Frenkel and J. Dorfman, Nature 126, 274 (1930).

${ }^{2}$ L. D. Landau and E. M. Lifschitz, "Electrodynamics of Continuous Media" (Pergamon Press, Oxford, England, 1984).

${ }^{3}$ C. G. Montgomery, Phys. Rev. 38, 1782 (1931).

${ }^{4}$ W. C. Elmore, Phys. Rev. 54, 1092 (1938).

${ }^{5}$ C. P. Bean and J. D. Livingston, J. Appl. Phys. 30, $120 \mathrm{~S}$ (1959).

${ }^{6}$ C. P. Bean and I. S. Jacobs, J. Appl. Phys. 27, 1448 (1956); J. S. Jacobs and C. P. Bean, in Magnetism, edited by J. T. Rado and H. Suhl (Academic Press, New York, 1963), p. 271.
${ }^{7}$ W. Luo, S. R. Nagel, T. F. Rosenbaum and R. E. Rosensweig, Phys. Rev. Lett. 67, 2721 (1991).

${ }^{8}$ M. Hanson, C. Johansson, M. S. Pedersen and S. Morup,

- U.. Phys., Condens. Matter. 7, 9269 (1995).

$T_{10}^{9}$ J. A) Mydosh, J. Mag. Magn. Mater. 157/158, 606 (1996).

M. G. del Muro, X. Batlle, A. Labarta, J. M. Gonzalez and M. I. Montero, J. Appl. Phys. 81, 7427 (1997).

${ }^{11}$ K. S. Suslick, S.-B. Choe, A. A. Cichowlas, and M. W. Grinstaff, Nature 353, 414 (1991).

${ }^{12}$ X. Cao, Y. Koltypin, R. Prozorov, G. Kataby, I. Felner, and A. Gedanken, J. Mater. Res. 12, 402 (1997).

${ }^{13}$ T. Prozorov, R. Prozorov, Y. Koltypin, I. Felner, A. + MGsidalulen, J. Phys. Ghem. B 102, 10165 (1998).

${ }^{14}$ P. W. Anderson, Phys. Rev. Lett. 9, 309 (1962).

${ }^{15}$ M. R. Beasley, R. Labush, and W. W. Webb, Phys. Rev. 181, 682 (1969).

${ }^{16}$ Y. Yeshurun, A. P. Malozemoff and A. Shaulov, Rev. Mod. Phys. 68, 911 (1996).

${ }^{17}$ S. Morup and E. Tronc, Phys. Rev. Lett. 72, 3278 (1994).

${ }^{18}$ W. T. Coffey and D. S. F. Crothers, Phys. Rev. E 54, 4768 (1996).

19 J. E. Wegrowe, J. P. Meier, B. Doudin, J. P. Ansermet, W. Wernsdorfer, B. Barbara, W. T. Coffey, Y. P. Kalmykov and J. L. Dejardin, Europhys. Lett. 38, 329 (1997).

${ }^{20}$ C. Sanchez, J. M. Gonzalez-Miranda and J. Tejada, J. Mag. Magn. Mater. 140-144, 365 (1995).

${ }^{21}$ A. Aharoni, "Introduction to the theory of ferromagnetism" (Clarendon Press, Oxford), (1996).

22 S. Morup, M. B. Madsen, J. Franck, J. Villadsen and C. J. W. Koch, J. Mag. Magn. Mater. 40, 163 (1983).

23 S. Morup, Europhys. Lett. 28, 671 (1994).

${ }^{24}$ S. Bocquet, R. J. Pollard and J. D. Cashion, Phys. Rev. B 46, 11657 (1992).

${ }^{25}$ M. El-Hilo, K. O'Grady and R. W. Chantrell, J. Mag. Magn. Mater. 114, 307 (1992).

${ }^{26}$ T. Bitoh, K. Ohba, M. Takamatsu, T. Shirane and S. Chikazawa, J. Mag. Magn. Mater. 154, 59 (1996).

${ }^{27}$ W. T. Coffey and Y. P. Kalmykov, J. Mag. Magn. Mater. 164, 133 (1996).

${ }^{28}$ L. Balcells, O. Iglensias and A. Labarta, Physical Review B 55, 8940 (1997).

${ }^{29}$ M. G. del Muro, X. Batlle, A. Labarta, J. M. Gonzalez and M. I. Montero, J. Appl. Phys. 81, 3812 (1997).

${ }^{30}$ Y. L. Raikher and V. I. Stepanov, Phys. Rev. B 55, 15005 (1997).

${ }^{31}$ S. U. Jen, C. Y. Lee, Y. D. Yao and K. C. Lee, J. Mag. Magn. Mat. 96, 82 (1991).

${ }^{32}$ M. W. Grinstaff, M. B. Salamon and K. S. Suslik, Phys. Rev. B 48, 269 (1993).

${ }^{33}$ D. K. Lottis, R. M. White, E. Dan Dahlberg, Phys. Rev. Lett. 67, 362 (1991).

${ }^{34}$ R. D. Kirby, J. X. Shen, R. J. Hardy, D. J. Sellmyer, Phys. Rev. B 49, 10810 (1994); A. Liberatos, J. Earl, and R. W. Chantrell, Phys. Rev. B 53, 5493 (1996).

35 J. P. Bouchard and P. G. Zerah, Phys. Rev. B 47, 9095 (1993).

${ }^{36}$ M. A. Zaluska-Kotur and M. Cieplak, Europhys. Lett. 23, 85 (1993).

${ }^{37}$ L. Néel, Ann. Geophys. 5, 99 (1949).

38 S. Morup and G. Christiansen, J. Appl. Phys. 73, 6955 
(1993)

${ }^{39}$ S. Linderoth, L. Balcells, A. Labarta, J. Tejada, P. V. Hendriksen and S. A. Sethi, J. Mag. Magn. Mater. 124, 269 (1993).

${ }^{40}$ E. Viitala, J. Merikoski, M. Manninen and J. Timonen, Phys. Rev. B 55, 11541 (1997).

\section{FIGURE CAPTIONS}

Fig.1 X-ray diffraction patterns for (a) regular amorphous, (b) acicular amorphous and (c) crystalline samples.

Fig.2 Differential scanning colorimetry spectra for (a) regular amorphous, (b) acicular amorphous and (c) crystalline samples.

Fig.3 Transmission electron micrographs for (a) regular amorphous, (b) acicular amorphous and (c) crystalline samples.

Fig.4 Typical ZFC - FC curves with superimposed relaxation for the amorphous sample measured at $500 \mathrm{G}$ at different temperatures. Inset: full-range $\mathrm{ZFC}-\mathrm{FC}$ curve.

Fig.5 Typical relaxation curves measured in the amorphous sample in $500 G$ at different temperatures.

Fig.6 Normalized logarithmic relaxation rate $R$ for three types of samples as a function of temperature. Inset: $R$ as a function of a reduced temperature $T / T_{i r r}$.

Fig.7 Irreversibility lines for three types of samples.

Fig.8 Typical magnetization loops at $T=5 K$ (open circles) and at $T=100$ (solid line).

Fig.9 Magnetic relaxation at different values of magnetic field. Vertical lines are the $M(t)$ curves superimposed on a regular magnetization loop measured at the same temperature.

Fig.10 Normalized logarithmic relaxation rate $R$ for three samples as a function of magnetic field at $T=20 \mathrm{~K}$.

Fig.11 Schematic snapshots of magnetic moments distribution in powder sample at (a) beginning of the relaxation and (b) at latter time

Fig.12 Energy profiles after FC in magnetic high field $\left(H_{1}\right)$ and after reduction of the magnetic field, whence the relaxation starts $\left(H_{2}\right)$.

Fig.13 Energy profiles at the beginning and at the latter stage of the relaxation. Dots indicate population of magnetic moments of the particular energy minima.

Fig.14 Temperature dependence of energy $\Theta$ extracted from the measurements of normalized relaxation rate for the three samples.

Fig.15 Magnetic field dependence of the energy $\Theta$.

Fig. 16 Scaling of the $M(T)$ FC-ZFC curves with irreversibility temperature. 
Table I. Characteristic parameters of the samples

\begin{tabular}{||c|c|c||}
\hline \hline \multicolumn{1}{|c|}{ Sample } & $d[\mathrm{~nm}]$ & Surface area $\left[\mathrm{m}^{2} / \mathrm{g}\right]$ \\
\hline \hline regular amorphous & $\sim 50$ & 148 \\
\hline acicular amorphous & $\sim 5 \times 50$ & 164 \\
\hline crystalline & $\sim 200$ & 88 \\
\hline \hline
\end{tabular}


This figure "Slide1.JPG" is available in "JPG" format from: http://arxiv.org/ps/cond-mat/9811206v2 
This figure "Slide2.JPG" is available in "JPG" format from: http://arxiv.org/ps/cond-mat/9811206v2 
This figure "Slide3.JPG" is available in "JPG" format from: http://arxiv.org/ps/cond-mat/9811206v2 
This figure "Slide4.JPG" is available in "JPG" format from: http://arxiv.org/ps/cond-mat/9811206v2 
This figure "Slide5.JPG" is available in "JPG" format from: http://arxiv.org/ps/cond-mat/9811206v2 
This figure "Slide6.JPG" is available in "JPG" format from: http://arxiv.org/ps/cond-mat/9811206v2 
This figure "Slide7.JPG" is available in "JPG" format from: http://arxiv.org/ps/cond-mat/9811206v2 
This figure "Slide8.JPG" is available in "JPG" format from: http://arxiv.org/ps/cond-mat/9811206v2 
This figure "Slide9.JPG" is available in "JPG" format from: http://arxiv.org/ps/cond-mat/9811206v2 
This figure "Slide10.JPG" is available in "JPG" format from: http://arxiv.org/ps/cond-mat/9811206v2 
This figure "Slide11.JPG" is available in "JPG" format from: http://arxiv.org/ps/cond-mat/9811206v2 
This figure "Slide12.JPG" is available in "JPG" format from: http://arxiv.org/ps/cond-mat/9811206v2 
This figure "Slide13.JPG" is available in "JPG" format from: http://arxiv.org/ps/cond-mat/9811206v2 
This figure "Slide14.JPG" is available in "JPG" format from: http://arxiv.org/ps/cond-mat/9811206v2 
This figure "Slide15.JPG" is available in "JPG" format from: http://arxiv.org/ps/cond-mat/9811206v2 
This figure "Slide16.JPG" is available in "JPG" format from: http://arxiv.org/ps/cond-mat/9811206v2 